Research Article

\title{
Facial Emotion Recognition Predicts Alexithymia Using Machine Learning
}

\author{
Nima Farhoumandi $\mathbb{D}^{1},{ }^{1}$ Sadegh Mollaey $\mathbb{D D}^{1},{ }^{1}$ Soomaayeh Heysieattalab $\left(\mathbb{D},{ }^{2}\right.$ \\ Mostafa Zarean ${ }^{D},{ }^{1}$ and Reza Eyvazpour ${ }^{3}{ }^{3}$ \\ ${ }^{1}$ Department of Psychology, Faculty of Education and Psychology, University of Tabriz, Tabriz, Iran \\ ${ }^{2}$ Department of Cognitive Neuroscience, Faculty of Education and Psychology, University of Tabriz, Tabriz, Iran \\ ${ }^{3}$ Department of Biomedical Engineering, School of Electrical Engineering, Iran University of Science and Technology (IUST), \\ Tehran, Iran
}

Correspondence should be addressed to Soomaayeh Heysieattalab; s.heysiyattalab@tabrizu.ac.ir and Mostafa Zarean; zarean@ tabrizu.ac.ir

Received 11 May 2021; Revised 5 September 2021; Accepted 7 September 2021; Published 28 September 2021

Academic Editor: Abdelkader Nasreddine Belkacem

Copyright (c) 2021 Nima Farhoumandi et al. This is an open access article distributed under the Creative Commons Attribution License, which permits unrestricted use, distribution, and reproduction in any medium, provided the original work is properly cited.

Objective. Alexithymia, as a fundamental notion in the diagnosis of psychiatric disorders, is characterized by deficits in emotional processing and, consequently, difficulties in emotion recognition. Traditional tools for assessing alexithymia, which include interviews and self-report measures, have led to inconsistent results due to some limitations as insufficient insight. Therefore, the purpose of the present study was to propose a new screening tool that utilizes machine learning models based on the scores of facial emotion recognition task. Method. In a cross-sectional study, 55 students of the University of Tabriz were selected based on the inclusion and exclusion criteria and their scores in the Toronto Alexithymia Scale (TAS-20). Then, they completed the somatization subscale of Symptom Checklist-90 Revised (SCL-90-R), Beck Anxiety Inventory (BAI) and Beck Depression Inventory-II (BDI-II), and the facial emotion recognition (FER) task. Afterwards, support vector machine (SVM) and feedforward neural network (FNN) classifiers were implemented using K-fold cross validation to predict alexithymia, and the model performance was assessed with the area under the curve (AUC), accuracy, sensitivity, specificity, and F1-measure. Results. The models yielded an accuracy range of 72.7-81.8\% after feature selection and optimization. Our results suggested that ML models were able to accurately distinguish alexithymia and determine the most informative items for predicting alexithymia. Conclusion. Our results show that machine learning models using FER task, SCL-90-R, BDI-II, and BAI could successfully diagnose alexithymia and also represent the most influential factors of predicting it and can be used as a clinical instrument to help clinicians in diagnosis process and earlier detection of the disorder.

\section{Introduction}

Alexithymia could be briefly described as emotional blindness [1] and refers to the difficulty in expressing and recognizing emotional states [2]. Alexithymic individuals misinterpret the somatic symptoms of emotional arousal, try to express their emotional distress through physical complaints, and seek treatment for their physical symptoms [3]. It is also argued that alexithymic individuals exhibit difficulties in understanding and regulating their emotions [4].
The prevalence of alexithymia in the general population is $10 \%-13 \%$, and alexithymia symptoms are more prevalent among males [5].

Alexithymia, and particularly the subscale of "difficulty in identifying feelings," is associated with various psychiatric disorders [6-10]. For instance, follow-up studies show that chronic alexithymia is consistently associated with depression and various symptoms of psychological disorders such as cluster-c personality disorders [11-13]. Moreover, the presence of alexithymia predicts poorer treatment outcomes 
for anxiety and somatoform disorders [14], depression [15], alcoholism [16], and mixed psychiatric disorders [17]. Alexithymia also seems to be a common feature of neurological diseases, with most evidence available for patients with traumatic brain injury, stroke, and epilepsy [18]. Therefore, identification of alexithymia may have important preventive, diagnostic, and therapeutic implications.

Clinical judgment is the most common approach to assessing alexithymia, which is questionable in terms of psychometric quality and its unknown interrater reliability [19]. The next method involves the use of questionnaires, such as the Beth Israel Hospital Questionnaire (BIQ) and the Toronto Alexithymia Scale (TAS-20), which is inexpensive and can be administered more rapidly [20]. Using only selfreport tools and administering interviews have some limitations because the restricted introspection of subjects affects the results of the questionnaire [21], and interviews are more laborious to administer [20].

Over the recent years, clinicians have shown great interest in using analytical methods for efficiently diagnosing people with mental disorders from healthy individuals based on their scores. One of the methods used for this purpose is supervised machine learning (ML), which could automatically extract information from available data through creating different algorithms and techniques [22]. ML approaches are used in different areas, such as neuroimaging [22], malingering [23, 24], genetics [25], clinical medicine [26], and augmenting psychological tests [27]. The benefits of ML models in solving classification problems have been further proven [22]. Supervised ML methods allow for characterization at individual levels, thus yielding results with a potentially high level of clinical translation. Moreover, as inherently multivariate approaches, supervised ML methods are sensitive to spatially distributed and subtle effects in the brain, which would be undetectable if we were to use traditional univariate methods which focus on gross differences at the group level [22].

In the present study, we hypothesized that some common features of patients with alexithymia might be good predictors of alexithymia. Among these features, the distinct relationship of alexithymia with somatization, anxiety, and depression has also been a research priority $[2,5,11,28-31]$. A significant relationship has been reported between somatization and alexithymia in clinical and nonclinical samples [2]. It has been shown that individuals with alexithymia have aggravated depressive symptoms [32]. Other studies [29, 30] also support the hypothesis that the presence of alexithymia predisposes individuals to depression. Based on some evidence, anxiety or depression can trigger a reactive regression to emotional development, thereby developing alexithymic features [31].

Given the association of alexithymia with somatization, anxiety, and depression, it seems that the presence of any of the foregoing disorders can be indicative of alexithymia. Accordingly, it is assumed that some items of BDI-II, BAI, and the somatization subscale of SCL-90-R questionnaires can be used to predict alexithymia. Besides, alexithymic features within the context of defective emotion recognition and regulation could be considered as a potential risk factor for medical and psychiatric conditions $[33,34]$.

Facial emotion recognition (FER), as a process of identifying facial expressions, is one of the most important elements in social communications and interactions [35]. Facial expressions can represent our emotional states and affect the generation and regulation of emotional states and behaviors in response to environmental signals [36]. FER is impaired in patients with high levels of alexithymia [37-43]. In addition, alexithymic patients show less activity in areas of the brain involved in extracting emotional components from facial expressions and FER (amygdala, insula, inferior frontal gyrus, striatum, and middle temporal gyrus) $[27,38,43]$. Accordingly, given the role of alexithymia in psychopathology and psychiatry, as well as the association of alexithymia with FER defects, the present study aimed to investigate the relationship between FER deficits, somatization, depression, and anxiety with alexithymia levels. The level of alexithymia (TAS-20) was predicted using the FER task dataset and the somatization subscale of SCL-90-R, BDI-II, and BAI questionnaires, which had been implemented in the ML methods of the artificial neural network (ANN) and support vector machine (SVM).

\section{Materials and Methods}

2.1. Participants. The statistical population of this study included students of the University of Tabriz in the academic year 2019-2020. In the first phase of the study, a total of 388 students, 174 males (44.8\%) and 214 females (55.2\%), were selected via cluster sampling (age range $=19-35, M_{\text {age }}=23.27$, $\mathrm{SD}_{\text {age }}=3.93$ ). After receiving approval from the Local Ethics Committee (Department of Psychology, University of Tabriz), the students were informed about the aim of the research, their informed consent was obtained, and they completed the questionnaires. The inclusion criteria were age range of 19-35 and willingness to participate in the study. The exclusion criteria were age above 35 years or below 19 years, diagnosis of any psychiatric disorders, use of medications that affect attention or cognition, diagnosis of any medical illness, addiction to alcohol or other recreational drugs, and unwillingness to participate in the study.

In the second phase of the study, 26 participants (11 males and 15 females) who met the inclusion criteria were included in the experimental study. According to the inclusion and exclusion criteria, 29 age-matched healthy controls (including 6 males and 23 females) were selected. Scores above 60 (TAS > 60) were considered as participants with alexithymia and scores below that threshold were healthy controls (TAS $<60$ ) in the TAS-20 scale [44]. Table 1 provides a descriptive summary of the demographic data and questionnaires scores for all participants in the two groups (alexithymia and healthy control).

2.2. Behavioral Data. A total of 55 participants were included and divided into two groups (Alex and HC) based on the cut-off point on the TAS-20 scale. According to 
TABle 1: Descriptive statistics of demographic and questionnaire data for each group (Alex and HC), effect size (d), and results of Mann-Whitney $U$ test.

\begin{tabular}{|c|c|c|c|c|c|}
\hline & Alex $(N=26)$ & $\mathrm{HC}(N=29)$ & Effect size $(d)$ & Mann-Whitney $U$ test & Sig. \\
\hline Gender (male/female) & $11 / 15$ & $6 / 23$ & 0.47 & 295.50 & 0.086 \\
\hline Age $(M \pm S D)$ & $23.19 \pm 5.55$ & $23.965 \pm 5.69$ & 0.14 & 337.00 & 0.498 \\
\hline TAS-20 total score $(\mathrm{M} \pm \mathrm{SD})$ & $64.46 \pm 3.88$ & $34.689 \pm 2.80$ & -0.62 & .00 & 0.000 \\
\hline BDI-II-physical symp $(\mathrm{M} \pm \mathrm{SD})$ & $4.692 \pm 3.31$ & $1.413 \pm 1.50$ & -1.25 & 142.00 & 0.000 \\
\hline BDI-II-emotional symp $(\mathrm{M} \pm \mathrm{SD})$ & $5.80 \pm 4.13$ & $1.827 \pm 1.79$ & -1.22 & 148.00 & 0.000 \\
\hline BDI-II-cognitive symp $(\mathrm{M} \pm \mathrm{SD})$ & $6.07 \pm 4.38$ & $1.655 \pm 2.19$ & -1.25 & 130.00 & 0.000 \\
\hline $\mathrm{BAI}(\mathrm{M} \pm \mathrm{SD})$ & $12.96 \pm 9.02$ & $6.275 \pm 5.59$ & -0.88 & 186.00 & 0.001 \\
\hline Happy recognition $(\mathrm{M} \pm \mathrm{SD})$ & $0.99 \pm 0.00$ & $0.997 \pm 0.00$ & -0.06 & 353.50 & 0.379 \\
\hline Fear recognition $(\mathrm{M} \pm \mathrm{SD})$ & $0.74 \pm 0.16$ & $0.748 \pm 0.17$ & 0.001 & 368.00 & 0.879 \\
\hline Sadness recognition $(\mathrm{M} \pm \mathrm{SD})$ & $0.94 \pm 0.03$ & $0.939 \pm 0.06$ & -0.05 & 347.50 & 0.607 \\
\hline Anger recognition $(\mathrm{M} \pm \mathrm{SD})$ & $0.89 \pm 0.08$ & $0.879 \pm 0.13$ & -0.13 & 374.50 & 0.966 \\
\hline Neutral recognition $(\mathrm{M} \pm \mathrm{SD})$ & $0.98 \pm 0.02$ & $0.987 \pm 0.02$ & -0.06 & 360.50 & 0.715 \\
\hline Disgust recognition $(\mathrm{M} \pm \mathrm{SD})$ & $0.82 \pm 0.08$ & $0.853 \pm 0.11$ & 0.33 & 283.50 & 0.111 \\
\hline Surprise recognition $(\mathrm{M} \pm \mathrm{SD})$ & $0.98 \pm 0.02$ & $0.982 \pm 0.02$ & -0.12 & 341.50 & 0.454 \\
\hline Happy time $(\mathrm{M} \pm \mathrm{SD})$ & $2.26 \pm 0.34$ & $2.249 \pm 0.56$ & -0.03 & 302.00 & 0.206 \\
\hline Fear time $(\mathrm{M} \pm \mathrm{SD})$ & $2.90 \pm 0.57$ & $2.794 \pm 0.63$ & -0.19 & 293.00 & 0.157 \\
\hline Sadness time $(\mathrm{M} \pm \mathrm{SD})$ & $3.00 \pm 0.54$ & $2.941 \pm 0.55$ & -0.11 & 337.00 & 0.500 \\
\hline Anger time $(\mathrm{M} \pm \mathrm{SD})$ & $2.93 \pm 0.57$ & $2.741 \pm 0.53$ & -0.35 & 266.00 & 0.061 \\
\hline Neutral time $(M \pm S D)$ & $3.54 \pm 0.53$ & $3.356 \pm 0.63$ & -0.32 & 281.00 & 0.106 \\
\hline Disgust time $(\mathrm{M} \pm \mathrm{SD})$ & $2.70 \pm 0.64$ & $2.539 \pm 0.65$ & -0.25 & 298.00 & 0.183 \\
\hline Surprise time $(\mathrm{M} \pm \mathrm{SD})$ & $2.32 \pm 0.64$ & $2.419 \pm 0.62$ & 0.14 & 362.00 & 0.800 \\
\hline SCL-Q1 $(M \pm S D)$ & $1.15 \pm 1.08$ & $1.034 \pm 1.17$ & -0.010 & 336.50 & 0.470 \\
\hline SCL-Q2 $(M \pm S D)$ & $1.26 \pm 1.18$ & $0.862 \pm 0.95$ & -0.37 & 306.50 & 0.209 \\
\hline SCL-Q3 $(M \pm S D)$ & $0.653 \pm 0.97$ & $0.275 \pm 0.75$ & -0.43 & 300.00 & 0.080 \\
\hline SCL-Q4 $(\mathrm{M} \pm \mathrm{SD})$ & $1.07 \pm 1.09$ & $0.655 \pm 0.76$ & -0.44 & 301.50 & 0.172 \\
\hline SCL-Q5 $(\mathrm{M} \pm \mathrm{SD})$ & $0.57 \pm 1.06$ & $0.482 \pm 0.94$ & -0.09 & 371.50 & 0.906 \\
\hline SCL-Q6 $(\mathrm{M} \pm \mathrm{SD})$ & $1.65 \pm 1.35$ & $0.689 \pm 1.00$ & -0.80 & 215.00 & 0.004 \\
\hline SCL-Q7 $(\mathrm{M} \pm \mathrm{SD})$ & $0.23 \pm 0.42$ & $0.103 \pm 0.30$ & -0.33 & 329.00 & 0.207 \\
\hline SCL-Q8 $(\mathrm{M} \pm \mathrm{SD})$ & $0.76 \pm 1.06$ & $0.586 \pm 1.08$ & -0.17 & 324.50 & 0.308 \\
\hline SCL-Q9 $(\mathrm{M} \pm \mathrm{SD})$ & $0.92 \pm 1.19$ & $0.310 \pm 0.54$ & -0.65 & 279.50 & 0.055 \\
\hline SCL-Q10 $(\mathrm{M} \pm \mathrm{SD})$ & $0.53 \pm 1.06$ & $0.413 \pm 0.73$ & -0.13 & 373.00 & 0.933 \\
\hline SCL-Q11 $(\mathrm{M} \pm \mathrm{SD})$ & $1.30 \pm 1.15$ & $0.551 \pm 1.02$ & -0.69 & 225.00 & 0.005 \\
\hline SCL-Q12 $(\mathrm{M} \pm \mathrm{SD})$ & $0.84 \pm 1.12$ & $0.137 \pm 0.35$ & -0.83 & 253.50 & 0.008 \\
\hline
\end{tabular}

Note. M: mean; SD: standard deviation; TAS-20:Toronto alexithymia scale; BDI-II: Beck depression inventory; BAI: Beck anxiety inventory; SCL: somatization subscale of SCL-90-R.

this scaling, participants with a score above 60 (TAS $>60$ ) entered the alexithymia group and those scoring below 60 $($ TAS $<60)$ entered the healthy control group. Table 1 summarizes the subjects' clinical and demographic characteristics and shows the results of the Mann-Whitney $U$ test for each of the variables entered in the ML model. The results of the Chi-square test showed that the two groups were not significantly different in terms of gender distribution $\left(\chi^{2}=3.000, p=0.08\right)$.

\subsection{Instruments}

2.3.1. Somatization Subscale of the Symptom Checklist-90Revised (SCL-90-R). The questionnaire was primarily developed by Derogatis et al. [45], revised based on clinical experience and psychometric analysis, and finalized in 1976. Respondents had to answer 90 questions on a 5-point Likert scale (with 0 meaning "not at all" and 4 meaning "extremely"). The somatization subscale of the SCL-90-R is a 12 -item list of common somatic symptoms. It has good reliability in different studies [46]. Akhavan-Abiri and
Shaeiri [47] reported that the reliability of Cronbach's alpha coefficient was 0.87 in the student sample. The current study also indicated the reliability for this subscale with Cronbach's alpha of 0.86 .

2.3.2. Toronto Alexithymia Scale (TAS-20). The TAS is a 20item self-report questionnaire with three dimensions, namely, difficulty identifying feelings (DIF), difficulty describing feelings (DDF), and externally oriented thinking (EOT) [48]. Items are scored based on a 5-point Likert scale from strongly disagree (1) to strongly agree (5). The international cut-off values are as follows: $20-50=$ nonalexithymic subjects, $51-60=$ borderline alexithymic subjects, and $61-100=$ alexithymic subjects [44]. In our study, subjects with a total TAS score over 60 were considered as alexithymic, and those with a score under 60 were considered as nonalexithymic [33]. In the Iranian sample, the reliability of this questionnaire based on Cronbach's alpha was 0.79 for the whole TAS-20 scale and $0.75,0.71$, and 0.66 for DIF, DDF, and EOT, respectively. The total validities of the TAS-20 scale and 
DIF, DDF, and EOT dimensions in the Iranian clinical samples using test-retest were $0.77,0.73,0.69$, and 0.65 , respectively [49].

2.3.3. Beck Anxiety Inventory (BAI). Beck et al. [50] introduced the BAI which specifically measures the severity of clinical anxiety symptoms. The BAI is a 21 -item questionnaire in which participants are to select from four options that indicate the severity of anxiety. The four options for each question are scored on a 4-point Likert scale ranging from 0 to 3 . Its internal consistency coefficient was 0.92 , and its validity by a one-week test-retest was 0.75 [50]. In an Iranian population, Kaviani and Mousavi [51] reported a validity coefficient of 0.72 , a testretest reliability coefficient of 0.83 , and a Cronbach's alpha of 0.92 .

2.3.4. Beck Depression Inventory-Second Edition (BDI-II). Similar to its first edition, the questionnaire [52] comprises 21 items, where the individuals select one of the four options for each item that indicates the severity of their depression symptoms. Each item is also scored from 0 to 3 . The 21 items of the BDI-II are classified into three groups: affective, physical, and cognitive symptoms. Beck et al. [52] reported an internal consistency of 0.73 to 0.92 and an alpha coefficient of 0.86 for the patient group and 0.81 for the nonpatient group. Dobson and Mohammad Khani [53] obtained an alpha coefficient of 0.92 for the outpatients and 0.93 for the students, and a one-week test-retest coefficient of 0.93 was obtained in an Iranian sample.

2.3.5. Facial Emotion Recognition (FER) Task. The computer-based task is utilized to assess the recognition ability of facial emotional expressions. It was designed through the use of Python software and videos extracted from the Amsterdam Dynamic Facial Expression Set (ADFES), and it was validated by Hawk et al. [54].

The task starts with practice trials to allow the participants to become familiar with the main test. At the beginning of the experiment (Figure 1), a fixation cross was presented for $500 \mathrm{~ms}$ to locate the participants' visual attention. The practice section included 14 trials (videos of one male and one female presenter) and the main test included 168 trials (videos of 4 male and 4 female presenters with three random repetitions for each of the six basic emotions). Presenters with European and Asian faces presented each of the following expressions randomly: happiness, sadness, anger, disgust, fear, surprise, and no emotion. There was also a one-minute interval between the two stages of practice and the main test to eliminate the learning effect. In each section, videos were shown on a screen for 6 seconds. Participants were asked to press the space bar when they detected the emotional state first, and then they had 6 seconds to select the expressed emotion name, which was shown at the bottom of the screen.
2.4. Experimental Procedure. In the first phase of study, questionnaires were completed, and the participants were divided into the alexithymia group (called Alex) and healthy control group (called HC) based on the results of questionnaires. In the second phase, participants of both groups performed the FER task, which was conducted at the Emotion and Cognition Lab in the Department of Psychology, University of Tabriz. The characteristics of our sample (including the number of participants, mean, and standard deviation of our variable in each group) are presented in Table 1.

2.5. Classification Algorithms. The SVM and feedforward neural network (FNN) algorithms are two supervised learning classification methods that were used for classification in this study. SVM is a specific type of supervised ML method that aims to classify data points based on statistical learning theory through maximizing the margin between classes in a high-dimensional space [55-57]. An SVM classifier transforms the prediction problem into a square optimization problem, reducing the number of processes in the training phase and performing better and faster compared with other algorithms in terms of prediction accuracy $[57,58]$. The FNN algorithm uses multiple features to predict a target variable via learning input data through their weights. FNNs are also inspired by the biological neural system with features, such as parallel computing, nonlinearity, adaptability, responsiveness, and fault tolerance [59]. An FNN is made up of a number of layers (one-layer or multilayer designs) and a number of neurons with behaviors similar to a biological neuron. SVM and FNN classifiers are opted for owing to their accurate performance on nonlinear and high-dimensional data and their wide usage in a variety of different machine learning applications, including psychiatry and psychology research $[21,23,60]$.

2.6. Model Training and Performance Evaluation. Two sets of predictor variables were used to train each ML model: (1) all of the available data, namely, demographic information, SCL-90-R items, recognition, and time components of the FER task, and three subscales of depression and anxiety scores and (2) a subset of available data was selected based on the feature selection process, including SCL-5, neutral recognition, surprise recognition, fear time, disgust time, sadness time, anger time, depression physical symptoms, depression emotional symptoms, depression cognitive symptoms, and anxiety. The two sets of variables for training the ML models evaluate the necessity of all the predictor variables and determine the importance of the selected clinical scales during the feature selection process for classification between groups Alex and HC.

The $\mathrm{k}$-fold cross-validation $(k=5, k=10)$ technique was used to evaluate the generalizability of the models. This technique is typically used in ML approaches to compare and select a specific model for a given predictive modeling problem. In general, the estimations have a 


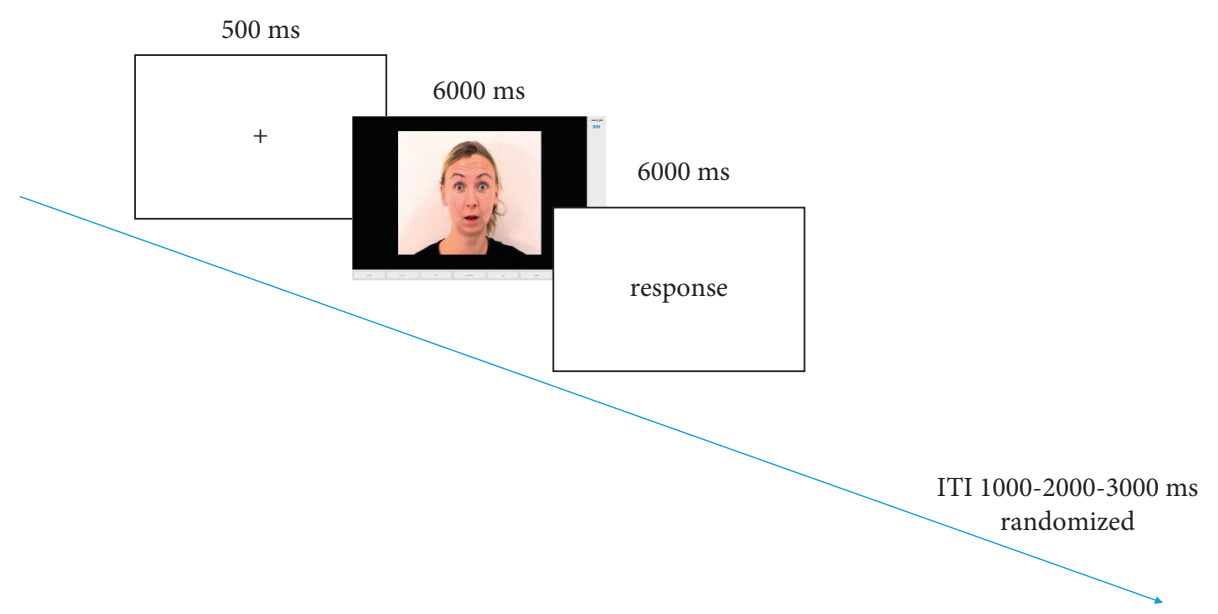

Figure 1: Facial emotion recognition (FER) task was used in this study. The total number of trials was 168 . A fixation cross, appearing on the screen for $500 \mathrm{~ms}$, was immediately followed by a dynamic facial expression presented for $6000 \mathrm{~ms}$. The participants had to press the space bar as soon as they recognized the emotion, and they had 6 seconds to choose the type of emotion from the options.

lower bias compared with other methods. Hyperparameter optimization was further adjusted using Bayesian optimization inside each cross-validation fold. This is an important procedure employed to classify new data as it allows the model to be simulated on unseen data. For SVM and FNN classifiers, the Kernel function and the number of layers and neurons are important hyperparameters, respectively; they are adjusted using $\mathrm{k}$-fold cross validation. Model performance was assessed using the area under the curve (AUC), accuracy, sensitivity, specificity, and F1-measure.

To select the best features for accurate prediction, a sequential (backward) feature selection method was used. In the backward elimination, the model started with all features and the least significant features were removed at each iteration, which improved the performance of the model. The process stopped when no more performance was observed.

For the final evaluation of the ML models, $20 \%$ of the total sample was conserved as a test set through random selection. The remaining $80 \%$ was used to train the model in $\mathrm{k}$-fold cross validation so as to optimize the model performance in every step. Afterwards, the final optimal classifiers were evaluated in the test set, which was not seen by the algorithm during the training procedure.

2.7. Confusion Matrix. In order to represent the classification results, the confusion matrix was used. To compare the performance of the classification model, the following measurements were conducted: accuracy, precision, specificity, and recall (sensitivity) calculated on the basis of confusion matrix, as well as the F1-measure calculated based on the harmonic means of precision and recall. The following equations show the relationships between the confusion matrix and the performance measurements (Figure 2):

$$
\begin{aligned}
\text { Accuracy } & =\frac{\mathrm{TP}}{\mathrm{TP}+\mathrm{TN}+\mathrm{FP}+\mathrm{FN}}, \\
\text { Precision } & =\frac{\mathrm{TP}}{\mathrm{TP}+\mathrm{FP}}, \\
\operatorname{Recal}(\text { sensitivity }) & =\frac{\mathrm{TP}}{\mathrm{TP}+\mathrm{FN}}, \\
\text { specificity } & =\frac{\mathrm{TN}}{\mathrm{TN}+\mathrm{FP}}, \\
F 1 \text { measure } & =\frac{2 * \text { Recal } * \text { Precision }}{\text { Recal }+ \text { Precision }}
\end{aligned}
$$

TP represents number of alexithymic patients detected correctly, TN represents number of healthy individuals detected correctly, FN represents number of alexithymic patients detected as healthy individuals, and FP represents number of healthy individuals detected as alexithymic patients.

2.8. Data Analysis. Data analysis was performed using SPSS and MATLAB v2017a. Of the 55 participants who were entered in the ML model, 29 individuals (6 males and 23 females with a mean age of $23.965(\mathrm{SD}=5.697))$ were placed in the HC group. Based on the cut-off point of the TAS-20 questionnaire, 26 individuals (11 males and 15 females with a mean age of $23.192(\mathrm{SD}=5.557))$ were assigned to the alexithymia group. Mann-Whitney $U$ test was performed to compare the clinical variables between the two groups (Alex and $\mathrm{HC}$ ); the effects sizes $(d)$ were specified through Borenstein's formula [61]. Cohen's suggested benchmarks (small: $d=0.2$, medium: $d=0.5$, and large: $d=0.8$ ) were also employed to interpret the magnitude of the effect sizes [62]. Furthermore, Chi-square test was utilized to compare categorical variables, such as gender.

As the first step of classification between the two groups, we ran classifiers including all the variables. In the second 
Confusion matrix

\begin{tabular}{|c|c|c|c|}
\hline & & \multicolumn{2}{|c|}{ Predicted } \\
\hline & & Negative & Positive \\
\hline \multirow{2}{*}{ Actual } & Negative & True Negative & False Positive \\
\hline & Positive & False Negative & True Positive \\
\hline
\end{tabular}

Figure 2: Confusion matrix. TN: True Negative, FP: False Positive, FN: False Negative, and TP: True Positive.

step, using the feature selection algorithm, the features increasing the classification accuracy were selected. The k-fold cross-validation technique was used to adjust the hyperparameters. Figure 3 depicts the whole process of model training, evaluation, and testing in this study.

\section{Results}

3.1. Classification Models. Both the SVM and FNN classification algorithms were able to distinguish between Alex and HC. The confusion matrix for the classification models is shown in Table 2. The confusion matrix shown in Table 2 represents the values of performance for the final classification model using two different classifiers (FNN and SVM), two different evaluation methods (using 5-fold cross validation and 10-fold cross validation) without/with feature selection and hyperparameter tuning. In general, confusion matrices represent the finding of the distribution of all the predicted values and how to compare with their true values.

Table 3 shows the model performance for each classification algorithm using the 5 -fold cross validation and 10 -fold cross validation with and without feature selection and hyperparameter tuning. The SVM model had a significantly better performance with feature selection and hyperparameter tuning. This classifier, trained by 10 -fold cross validation, performed better numerically, with a prediction accuracy of $81.8 \%$ and AUC of 0.80 . The performance of the FNN model did not change significantly (prediction accuracy of $72.7 \%$ and AUC of 0.73 ) when trained with feature selection and hyperparameter tuning or without optimization.

Hyperparameter optimization for the SVM algorithm was performed using Bayesian optimization. Radial basis function (RBF) Kernel was selected to define the SVM model and the optimum hyperparameters for this model $=893.56$ and Kernel scale $=6.03$. Regarding the feedforward neural network, the settings included one hidden layer and 10 neurons. These hyperparameters are considered as the default for the simplest neural network.

Through the feature selection process, we also found the 11 most important predictors that would differentiate between the Alex and HC groups. These predictors were SCL-5, neutral recognition, surprise recognition, fear time, disgust time, sadness time, anger time, depression physical symptoms, depression emotional symptoms, depression cognitive symptoms, and anxiety. In order to specify the effect of optimization and feature selection on models, the classifiers were run with and without feature selection.

\section{Discussion}

The recent years have seen an increase in the use of new methods such as ML to diagnose a variety of medical diseases and psychiatric disorders; these approaches can replace tools, such as clinical judgment and questionnaires. In addition, the existing methods have some disadvantages that make it difficult to diagnose these conditions with more certainty. For instance, Mannarini et al. [20] showed the downsides of using TAS-20 and Toronto Structured Interview for Alexithymia (TSIA). Their findings indicated that TSIA was more time-consuming (minimum $40 \mathrm{~min}$ ) and required training to be administered, and its accuracy was dependent on standardized administration and rating across different interviewers. The accuracy of the TAS-20 results also depends on the respondents' motivation and ability to reply sincerely [20]. Furthermore, the common statistical methods cannot diagnose disorders based on the existing data [21]. In contrast, ML models are based on feature selection to detect the optimum predictors of the disorders [22].

The objective of the present study was to use the most appropriate ML model capable of making the best distinction between alexithymic and healthy individuals. Previously, two studies predicted alexithymia utilizing machine learning models. Yöntem and Adem [63] applied an SVM model to predict the levels of alexithymia through automatic thoughts dataset. Their findings suggest that automatic thoughts would be helpful in the prediction of alexithymia. Orrù et al. [21] developed a machine learning model which increases the diagnosticity of alexithymia in patients with fibromyalgia. However, these studies predicted alexithymia through questionnaire scores and they did not utilize other neuropsychological measurements. In the present study, we implemented ML approach to predict alexithymia based on the FER task. Our findings support the hypothesis that utilizing $\mathrm{ML}$ techniques increases the ability to detect alexithymia through FER scores. As explained in the Results section, the traditional statistical analysis showed that most variables and items in this study had a significant range of effects on alexithymia.

On the other hand, classification models based on $\mathrm{ML}$ techniques had a higher accuracy in the range of 72.7-81.8\% following feature selection and optimization. The classifier was able to (i) correctly classify the subjects into two groups (Alex and HC) and (ii) identify the most informative features and predictors. The findings of the current study suggested that the SVM model using 10 -fold cross validation and feature selection yielded an accuracy of $81.8 \%$ 


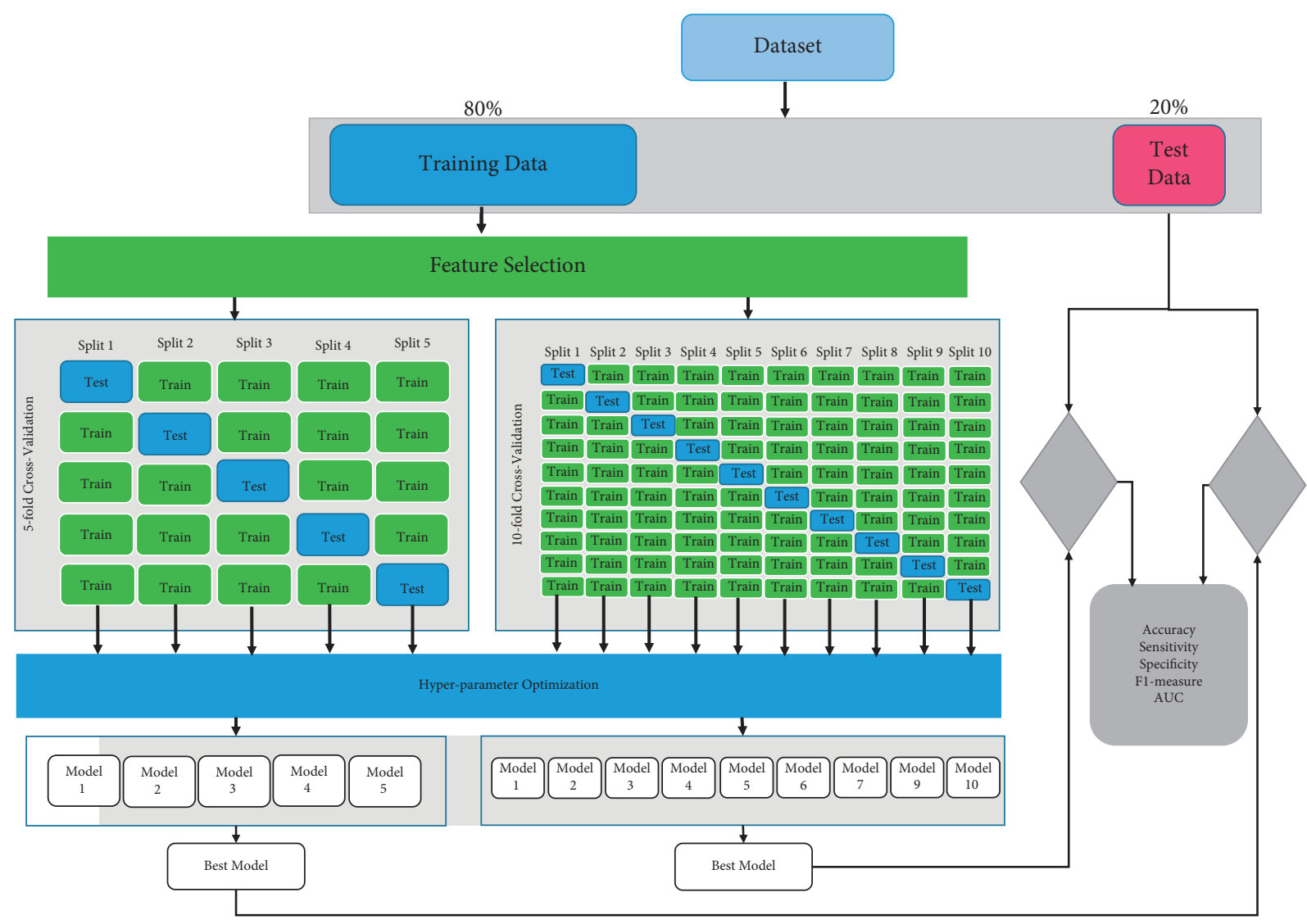

FIgURE 3: Machine learning procedure for training and testing the data.

TABLE 2: Confusion matrix of the final model by two different classifiers (FNN and SVM), two different evaluation methods (using 5-fold cross validation and 10 -fold cross validation) without/with feature selection and hyperparameter tuning.

\begin{tabular}{|c|c|c|c|c|c|c|}
\hline & & & \multicolumn{4}{|c|}{ Predicted } \\
\hline & & & \multicolumn{2}{|c|}{$\begin{array}{c}\text { Without feature selection and } \\
\text { optimization }\end{array}$} & \multicolumn{2}{|c|}{$\begin{array}{c}\text { With feature selection and } \\
\text { optimization }\end{array}$} \\
\hline & & & Negative & Positive & Negative & Positive \\
\hline \multirow{10}{*}{ Actual } & \multicolumn{6}{|c|}{ 10-Fold cross validation } \\
\hline & \multirow{2}{*}{ SVM } & Negative & 4 & 1 & 3 & 1 \\
\hline & & Positive & 3 & 3 & 1 & 6 \\
\hline & \multirow{2}{*}{ FNN } & Negative & 2 & 2 & 3 & 3 \\
\hline & & Positive & 2 & 5 & 1 & 4 \\
\hline & \multicolumn{6}{|c|}{ 5-Fold cross validation } \\
\hline & \multirow{2}{*}{ SVM } & Negative & 4 & 0 & 3 & 1 \\
\hline & & Positive & 4 & 3 & 2 & 5 \\
\hline & \multirow{2}{*}{ FNN } & Negative & 2 & 3 & 2 & 3 \\
\hline & & Positive & 2 & 4 & 2 & 4 \\
\hline
\end{tabular}

Note. TP represents number of alexithymic patients detected correctly, TN represents number of healthy individuals detected correctly, FN represents number of alexithymic patients detected as healthy individuals, and FP represents number of healthy individuals detected as alexithymic patients.

(AUC $=0.8, F 1=0.84)$. According to the literature, all of the most important predictors selected using the feature selection method are associated with one another; using a combination of these items in our measures will enable mental health professionals to predict alexithymia more accurately. Therefore, based on our findings, we are going to design a gadget that can help the assistants of psychologists or psychiatrists in clinical settings to (1) predict alexithymia without the need for prior training and (2) interpret the results without them being influenced by the administrator's bias.

As mentioned in the Introduction, alexithymia has relatively high prevalence in societies, is associated with a variety of psychological disorders, and reduces the effectiveness of treatment in a number of mental disorders. In addition, patients are likely to misinterpret their emotional arousal as a sign of disease [64]. This can lead to an overperception of the disease and patients seeking medical help, 
TABle 3: Model measurements including accuracy, sensitivity, specificity, AUC, and F1-measure by two different classifiers (FNN and SVM), two different evaluation methods (using 5-fold cross validation and 10-fold cross validation) without/with feature selection and hyperparameter tuning.

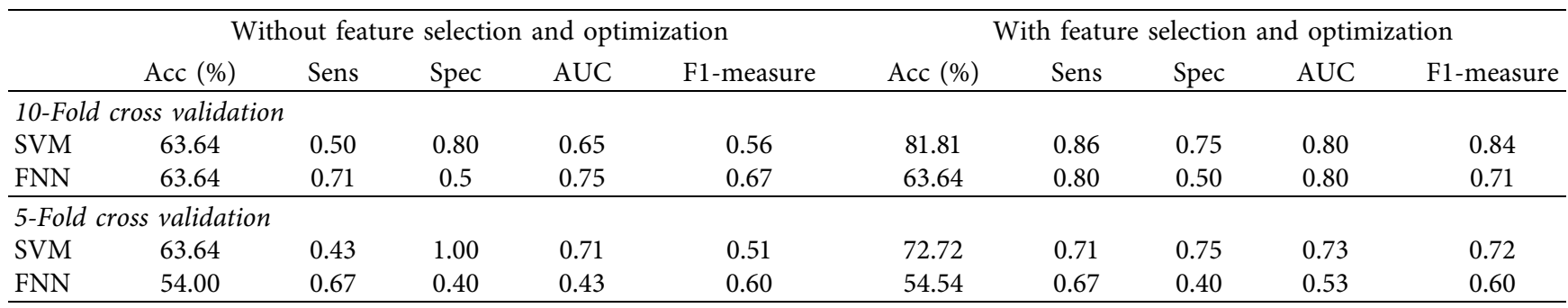

Note. AUC stands for area under the curve in ROC analysis and F1-measure. FNN, feedforward neural network; SVM, support vector machine; Acc, accuracy; Sens, sensitivity; Spec, specificity.

while there is no medical explanation for these symptoms [21]. Accordingly, improving the diagnostic methods of alexithymia, such as using ML models, can help therapists identify and present a professional, timely treatment and enhance the quality of life in these patients. The results might highlight the difficulty in identifying and detecting emotions, such as apathy and surprise and their reaction time in recognizing such emotions as fear, disgust, sadness, and anger. Among these, the recognition of neutral emotional states was notable. These findings could provide important implications for future research that should investigate the factors involved in the problems of alexithymic patients.

There were some limitations in this study. Our patients were not diagnosed according to a structured diagnostic interview; rather they were scored based on their selfreport. The second limitation was the small size of our sample. Of the 388 participants who responded to the TAS, only 26 persons received a definite cut-off point for the diagnosis of alexithymia. Future replications with a larger sample size are necessary for more definitive conclusions. Besides, regarding the conflicting literature about gender differences in the prevalence of alexithymia, it is recommended that future studies should consider the role of gender in their results.

\section{Conclusion}

We used a combination of questionnaire items (somatization subscale from SCL-90-R, BDI-II, and BAI) and FER task to predict alexithymia, and these measurements complement each other and increase the prediction accuracy. Also, using our proposed gadget, the accuracy of the diagnosis (done by either the therapist or assistant therapist) increases and remains unaffected by factors such as assessor's bias and the difficulty in interpreting the result of interviews or questionnaires, and there is no need for prior training to interpret the findings of our gadget. Besides, one of the common problems in using questionnaires is that the subjects (patients) probably try to provide a positive image of themselves. In the FER task, however, this problem disappears due to the nature of the test that one only requires to choose the right emotion from the available options.

\section{Data Availability}

The raw data used in this study will be made available by the authors to other qualified researchers.

\section{Ethical Approval}

All procedures performed in this study, involving human participants, were in accordance with the ethical standards of the institutional research committee and the 1964 Helsinki Declaration and its later amendments or comparable ethical standards.

\section{Consent}

All participants, to whom the authors are thankful, were adults and consented to participating in the research.

\section{Conflicts of Interest}

The authors have no conflicts of interest to declare.

\section{Authors' Contributions}

Nima Farhoumandi contributed to resources and data curation and wrote and prepared the original draft. Sadegh Mollaey contributed to software and formal analysis. Soomayeh Heysieattalab conceptualized, validated, and supervised the study, developed methodology, and wrote and prepared the original draft. Mostafa Zarean conceptualized and validated the study and developed the methodology. Reza Eyvazpour edited the manuscript and visualized the study.

\section{References}

[1] H. J. Grabe, C. Spitzer, and H. J. Freyberger, “Alexithymia and personality in relation to dimensions of psychopathology," American Journal of Psychiatry, vol. 161, no. 7, pp. 1299-1301, 2004.

[2] A. K. Mattila, E. Kronholm, A. Jula et al., "Alexithymia and somatization in general population," Psychosomatic Medicine, vol. 70, no. 6, pp. 716-722, 2008.

[3] R. M. Bagby, G. J. Taylor, and J. D. Parker, "The nomological domain of the alexithymia construct," The (Non) Expression of 
Emotions in Health and Disease, pp. 95-102, Tilburg University, Tilburg, Netherlands, 1997.

[4] I. Motan and T. Gençöz, "The relationship between the dimensions of alexithymia and the intensity of depression and anxiety," Turkish Journal of Psychiatry, vol. 18, no. 4, 2007.

[5] E. Dalbudak, C. Evren, S. Aldemir, K. S. Coskun, F. G. Yıldırım, and H. Ugurlu, "Alexithymia and personality in relation to social anxiety among university students," Psychiatry Research, vol. 209, no. 2, pp. 167-172, 2013.

[6] S. Berthoz, F. Perdereau, N. Godart, M. Corcos, and M. G. Haviland, "Observer- and self-rated alexithymia in eating disorder patients: levels and correspondence among three measures," Journal of Psychosomatic Research, vol. 62, no. 3, pp. 341-347, 2007.

[7] G. Coriale, E. Bilotta, L. Leone et al., "Avoidance coping strategies, alexithymia and alcohol abuse: a mediation analysis," Addictive Behaviors, vol. 37, no. 11, pp. 1224-1229, 2012.

[8] F. L. Coolidge, A. J. Estey, D. L. Segal, and P. D. Marle, "Are alexithymia and schizoid personality disorder synonymous diagnoses?" Comprehensive Psychiatry, vol. 54, no. 2, pp. 141-148, 2013.

[9] F. Declercq, S. Vanheule, and J. Deheegher, "Alexithymia and posttraumatic stress: subscales and symptom clusters," Journal of Clinical Psychology, vol. 66, no. 10, pp. 1076-1089, 2010.

[10] K. Honkalampi, J. Hintikka, P. Saarinen, J. Lehtonen, and H. Viinamäki, "Is alexithymia a permanent feature in depressed patients?" Psychotherapy and Psychosomatics, vol. 69, no. 6, pp. 303-308, 2000.

[11] B. Bankier, M. Aigner, and M. Bach, "Alexithymia in DSM-IV disorder: comparative evaluation of somatoform disorder, panic disorder, obsessive-compulsive disorder, and depression," Psychosomatics, vol. 42, no. 3, pp. 235-240, 2001.

[12] K. Honkalampi, J. Hintikka, R. Antikainen, J. Lehtonen, and H. Viinamäki, "Alexithymia in patients with major depressive disorder and comorbid cluster $\mathrm{C}$ personality disorders: a 6month follow-up study," Journal of Personality Disorders, vol. 15, no. 3, pp. 245-254, 2001.

[13] C. Marchesi, S. Fontò, C. Balista, C. Cimmino, and C. Maggini, "Relationship between alexithymia and panic disorder: a longitudinal study to answer an open question," Psychotherapy and Psychosomatics, vol. 74, no. 1, pp. 56-60, 2005.

[14] M. Bach and D. Bach, "Predictive value of alexithymia: a prospective study in somatizing patients," Psychotherapy and Psychosomatics, vol. 64, no. 1, pp. 43-48, 1995.

[15] J. S. Ogrodniczuk, W. E. Piper, and A. S. Joyce, "Alexithymia as a predictor of residual symptoms in depressed patients who respond to short-term psychotherapy," American Journal of Psychotherapy, vol. 58, no. 2, pp. 150-161, 2004.

[16] C. Cleland, S. Magura, J. Foote, A. Rosenblum, and N. Kosanke, "Psychometric properties of the Toronto alexithymia scale (TAS-20) for substance users," Journal of Psychosomatic Research, vol. 58, no. 3, pp. 299-306, 2005.

[17] M. McCallum, W. E. Piper, J. S. Ogrodniczuk, and A. S. Joyce, "Relationships among psychological mindedness, alexithymia and outcome in four forms of short-term psychotherapy," Psychology and Psychotherapy: Theory, Research and Practice, vol. 76, no. 2, pp. 133-144, 2003.

[18] L. Ricciardi, B. Demartini, A. Fotopoulou, and M. J. Edwards, "Alexithymia in neurological disease: a review," Journal of Neuropsychiatry and Clinical Neurosciences, vol. 27, no. 3, pp. 179-187, 2015.

[19] M. A. Lumley, L. C. Neely, and A. J. Burger, "The assessment of alexithymia in medical settings: implications for understanding and treating health problems," Journal of Personality Assessment, vol. 89, no. 3, pp. 230-246, 2007.

[20] S. Mannarini, R. Nacinovich, M. Bomba, and L. Balottin, "Alexithymia in parents and adolescent anorexic daughters: comparing the responses to TSIA and TAS-20 scales," Neuropsychiatric Disease and Treatment, vol. 10, p. 1941, 2014.

[21] G. Orrù, A. Gemignani, R. Ciacchini, L. Bazzichi, and C. Conversano, "Machine learning increases diagnosticity in psychometric evaluation of alexithymia in fibromyalgia," Frontiers of Medicine, vol. 6, p. 319, 2020.

[22] G. Orrù, W. Pettersson-Yeo, A. F. Marquand, G. Sartori, and A. Mechelli, "Using support vector machine to identify imaging biomarkers of neurological and psychiatric disease: a critical review," Neuroscience \& Biobehavioral Reviews, vol. 36, no. 4, pp. 1140-1152, 2012.

[23] C. Mazza, M. Monaro, G. Orrù et al., "Introducing machine learning to detect personality faking-good in a male sample: a new model based on Minnesota multiphasic personality inventory-2 restructured form scales and reaction times," Frontiers in Psychiatry, vol. 10, p. 389, 2019.

[24] G. Pace, G. Orrù, M. Monaro et al., "Malingering detection of cognitive impairment with the B test is boosted using machine learning," Frontiers in Psychology, vol. 10, p. 1650, 2019.

[25] M. W. Libbrecht and W. S. Noble, "Machine learning applications in genetics and genomics," Nature Reviews Genetics, vol. 16, no. 6, pp. 321-332, 2015.

[26] Z. Obermeyer and E. J. Emanuel, "Predicting the future - big data, machine learning, and clinical medicine," New England Journal of Medicine, vol. 375, no. 13, pp. 1216-1219, 2016.

[27] J. V. Haxby, E. A. Hoffman, and M. I. Gobbini, "The distributed human neural system for face perception," Trends in Cognitive Sciences, vol. 4, no. 6, pp. 223-233, 2000.

[28] M. Karukivi, L. Hautala, O. Kaleva et al., "Alexithymia is associated with anxiety among adolescents," Journal of Affective Disorders, vol. 125, no. 1-3, pp. 383-387, 2010.

[29] M. Gilanifar and M. A. Delavar, "Alexithymia in pregnant women: it's relationship with depression," ASEAN Journal of Psychiatry, vol. 17, no. 1, 2016.

[30] L. Hemming, G. Haddock, J. Shaw, and D. Pratt, "Alexithymia and its associations with depression, suicidality, and aggression: an overview of the literature," Frontiers in Psychiatry, vol. 10, p. 203, 2019.

[31] K. Honkalampi, J. Hintikka, A. Tanskanen, J. Lehtonen, and H. Viinamäki, "Depression is strongly associated with alexithymia in the general population," Journal of Psychosomatic Research, vol. 48, no. 1, pp. 99-104, 2000.

[32] J. Guidi, G. A. Fava, A. Picardi et al., "Subtyping depression in the medically ill by cluster analysis," Journal of Affective Disorders, vol. 132, no. 3, pp. 383-388, 2011.

[33] G. J. Taylor, R. M. Bagby, and J. D. Parker, Disorders of Affect Regulation: Alexithymia in Medical and Psychiatric Illness, Cambridge University Press, Cambridge, UK, 1999.

[34] G. J. Taylor and R. M. Bagby, "New trends in alexithymia research," Psychotherapy and Psychosomatics, vol. 73, no. 2, pp. 68-77, 2004.

[35] L. R. Demenescu, R. Kortekaas, J. A. den Boer, and A. Aleman, "Impaired attribution of emotion to facial expressions in anxiety and major depression," PloS One, vol. 5, no. 12, Article ID e15058, 2010.

[36] M. L. Phillips, W. C. Drevets, S. L. Rauch, and R. Lane, "Neurobiology of emotion perception I: the neural basis of normal emotion perception," Biological Psychiatry, vol. 54, no. 5, pp. 504-514, 2003. 
[37] R. Brewer, R. Cook, V. Cardi, J. Treasure, and G. Bird, "Emotion recognition deficits in eating disorders are explained by co-occurring alexithymia," Royal Society Open Science, vol. 2, no. 1, Article ID 140382, 2015.

[38] S. Jongen, N. Axmacher, N. A. W. Kremers et al., "An investigation of facial emotion recognition impairments in alexithymia and its neural correlates," Behavioural Brain Research, vol. 271, pp. 129-139, 2014.

[39] R. D. Lane, L. Sechrest, R. Riedel, D. E. Shapiro, and A. W. Kaszniak, "Pervasive emotion recognition deficit common to alexithymia and the repressive coping style," Psychosomatic Medicine, vol. 62, no. 4, pp. 492-501, 2000.

[40] J. D. Parker, G. J. Taylor, and R. M. Bagby, "Alexithymia and the recognition of facial expressions of emotion," Psychotherapy and Psychosomatics, vol. 59, no. 3-4, pp. 197-202, 1993.

[41] C. Subic-Wrana, M. E. Beutel, A. Knebel, and R. D. Lane, "Theory of mind and emotional awareness deficits in patients with somatoform disorders," Psychosomatic Medicine, vol. 72, no. 4, pp. 404-411, 2010.

[42] V. Venugopalan, M. Elkal, R. V. Behere, S. K. Praharaj, and H. Kanaradi, "A study on alexithymia, quality of life, and facial emotion recognition abilities in somatoform disorders," Journal of Postgraduate Medicine Education and Research, vol. 52, no. 3, pp. 110-116, 2018.

[43] M. I. Gobbini and J. V. Haxby, "Neural systems for recognition of familiar faces," Neuropsychologia, vol. 45, no. 1 , pp. 32-41, 2007.

[44] C. Bressi, G. Taylor, J. Parker et al., "Cross validation of the factor structure of the 20-item Toronto Alexithymia Scale: an Italian multicenter study," Journal of Psychosomatic Research, vol. 41, no. 6, pp. 551-559, 1996.

[45] L. R. Derogatis and P. A. Cleary, "Confirmation of the dimensional structure of the scl-90: a study in construct validation," Journal of Clinical Psychology, vol. 33, no. 4, pp. 981-989, 1977.

[46] K. Sayar, L. J. Kirmayer, and S. S. Taillefer, "Predictors of somatic symptoms in depressive disorder," General Hospital Psychiatry, vol. 25, no. 2, pp. 108-114, 2003.

[47] F. Akhavan Abiri and M. Shairi, "Validity and reliability of symptom checklist-90-revised (SCL-90-R) and brief symptom inventory-53 (BSI-53)," Clinical Psychology and Personality, vol. 17, no. 2, pp. 169-195, 2020.

[48] J. Dere, C. F. Falk, and A. G. Ryder, "Unpacking cultural differences in alexithymia," Journal of Cross-Cultural Psychology, vol. 43, no. 8, pp. 1297-1312, 2012.

[49] M. A. Besharat, "Relationship of Emotional insufficiency with anxiety, depression, psychological helplessness, and wellbeing," Quarterly of Psychological Novel Studies, pp. 17-40, 1993.

[50] A. T. Beck, N. Epstein, G. Brown, and R. A. Steer, "An inventory for measuring clinical anxiety: psychometric properties," Journal of Consulting and Clinical Psychology, vol. 56, no. 6, pp. 893-897, 1988.

[51] H. Kaviani and A. S. Mousavi, "Psychometric properties of the persian version of beck anxiety inventory (bai)," Tehran University Medical Journal, vol. 66, no. 2, pp. 136-140, 2008.

[52] A. T. Beck, R. A. Steer, and G. K. Brown, Beck Depression Inventory Fast Scree for Medical Patients, Psychological Corporation, San Antonio, TX, USA, 2000.

[53] K. S. Dabson and P. Mohammad Khani, "Psychometric characteristics of Beck depression inventory - II in patients with major depressive disorder," Archives of Rehabilitation (Journal of Rehabilitation), vol. 29, no. 1, pp. 82-88, 2007.
[54] S. T. Hawk, J. Van der Schalk, and A. H. Fischer, "Moving faces, looking places: the amsterdam dynamic facial expressions set (adfes)," in Proceedings of the 12th European Conference on Facial Expressions, vol. 4, Geneva, Switzerland, September 2008.

[55] F. Pereira, T. Mitchell, and M. Botvinick, "Machine learning classifiers and fMRI: a tutorial overview," NeuroImage, vol. 45, no. 1, pp. S199-S209, 2009.

[56] V. N. Vapnik, The Nature of Statistical Learning Theory, the Nature of Statistical Learning Theory, Springer, Berlin, Germany, 1995.

[57] V. N. Vapnik, The Nature of Statistical Learning Theory, Springer, Berlin, Germany, 2000.

[58] I. Nitze, U. Schulthess, and H. Asche, "Comparison of machine learning algorithms random forest, artificial neural network and support vector machine to maximum likelihood for supervised crop type classification," in Proceedings of the 4th Conference on GEographic Object-Based Image Analysis, p. 35, Rio de Janeiro, Brazil, May 2012.

[59] S. Haykins, Neural Networks: A Comprehensive Foundation, Prentice Hall PTR, Hoboken, NJ, USA, 1st edition, 1994.

[60] G. Briganti, M. Scutari, and P. Linkowski, "A machine learning approach to relationships among alexithymia components," Psychiatria Danubina, vol. 32, no. Suppl 1, pp. 180-187, 2020.

[61] M. Borenstein, H. Cooper, L. Hedges, and J. Valentine, "Effect sizes for continuous data," The Handbook of Research Synthesis and Meta-Analysis, vol. 2, pp. 221-235, Russell Sage Foundation, New York, NY, USA, 2009.

[62] D. Lakens, "Calculating and reporting effect sizes to facilitate cumulative science: a practical primer for t-tests and ANOVAs," Frontiers in Psychology, vol. 4, p. 863, 2013.

[63] M. K. Yöntem and K. Adem, "Prediction of the level of alexithymia through machine learning methods applied to automatic thoughts," Current Approaches in Psychiatry/Psikiyatride Guncel Yaklasimlar, vol. 11, pp. 64-78, 2019.

[64] M. A. Lumley, L. Stettner, and F. Wehmer, "How are alexithymia and physical illness linked? A review and critique of pathways," Journal of Psychosomatic Research, vol. 41, no. 6, pp. 505-518, 1996. 